\title{
Medicarpin induces lipolysis via activation of Protein Kinase A in brown adipocytes
}

\author{
Khan Mohammad Imran ${ }^{1,2}$, Dahyeon Yoon ${ }^{1,2}$, Tae-Jin Lee ${ }^{3}$ \& Yong-Sik Kim ${ }^{1,2, *}$ \\ ${ }^{1}$ Institute of tissue regeneration, College of Medicine, Soonchunhyang University, ${ }^{2}$ Department of Microbiology, College of Medicine, \\ Soonchunhyang University, Cheonan 31151, ${ }^{3}$ Department of Anatomy, College of Medicine, Yeungnam University, Daegu 42415, Korea
}

\begin{abstract}
Natural pterocarpan Medicarpin (Med) has been shown to have various beneficial biological roles, including inhibition of osteoclastogenesis, stimulation of bone regeneration and induction of apoptosis. However, the effect of the Med on lipolysis in adipocytes has not been reported. Here, we show the effect of Med on lipolysis in different mouse adipocytes and elucidate the underlying mechanism. We observed that Med treatment promoted release of glycerol in the media. Differentiated mouse brown adipose tissue cells were treated with Med. RNA-Seq analysis was performed to elucidate the effect of med and subsequently was confirmed by qRT-PCR and western blotting analyses. Med treatment increased both protein and gene expression levels of hormone-sensitive lipase (Hsl) and adipose triglyceride lipase (Atgl), which are two critical enzymes necessary for lipolysis. Mechanistic study showed that Med activates Protein Kinase A (PKA) and phosphorylates $\mathrm{Hsl}$ at PKA target position at Serine ${ }^{660}$. Silencing of PKA gene by short interfering RNA attenuated the Med-induced increase in glycerol release and Hsl phosphorylation. The results unveil that Med boosts lipolysis via a PKA-dependent pathway in adipocytes and may provide a possible avenue of further research of Med mediated reduction of body fat. [BMB Reports 2018; 51(5): 249-254]
\end{abstract}

\section{INTRODUCTION}

Adipocytes regulate fundamental physiological processes of storing and mobilizing energy. Energy imbalance towards excess consumption of energy rich food is mostly stored as fat in adipose tissue, and is released through carefully regulated processes of lipolysis upon stimulation (1). Balanced adipose

${ }^{*}$ Corresponding author. Tel: +82-41-570-2413; Fax: +82-41-5752412; E-mail: yongsikkim@sch.ac.kr

https://doi.org/10.5483/BMBRep.2018.51.5.228

Received 29 November 2017, Revised 19 December 2017, Accepted 18 January 2018

Keywords: Brown Adipose Tissue, Lipolysis, Medicarpin, Protein Kinase A, RNA-Seq cell function is crucial to control whole-body lipid and glucose metabolism, and any alteration to this process is associated with numerous metabolic disorders such as obesity and diabetes (2).

$\beta$-adrenergic receptors initiate lipolysis activation cascade by the activation of the CAMP-dependent PKA through the stimulation of CAMP production. Protein Kinase A (PKA) then phosphorylates $\mathrm{Hsl}$ at $\mathrm{Ser}^{563}$, Ser ${ }^{659}$, and $\mathrm{Ser}^{660}$ positions and activates lipolysis $(3,4)$. Hsl's intrinsic activity is increased by the PKA-dependent phosphorylation and promotes Hsl's access to TAG (4).

Brown and white fat possess contrasting functions. Recent discovery of functional brown adipose tissue in adult human and its physiological significance have turned attention to this fat depot as an anti-obesity target $(5,6)$. Studies have also revealed that lipolysis is necessary for the proton conductance of brown-fat mitochondria i.e. proper uncoupling of mitochondrial oxidative reaction (7-9).

Hormone sensitive lipase $(\mathrm{Hsl})$ and adipose triglyceride lipase (Atgl) possess capacity to the first ester bond of Triacylglycerol (TAG) by hydrolysis and of which deletion impairs lipid metabolism $(4,10,11)$. Translocation of $\mathrm{Hsl}$ from a cytosol to the surface of the lipid droplet is an important step in lipolysis activation. Hsl travels to the surface of lipid droplets from the cytosol and interacts with perilipin and neutral lipids. Notable, perilipin knockout adipocytes are incapable of translocating $\mathrm{Hsl}$ and thus lipolysis is hampered (12). In lipid droplet, perilipin acts as a coordinator of enzymes in response to the metabolic state of the adipocyte. Hence, lipase access is limited by the action of perilipin in normal conditions $(4,12)$.

In this study, we sought to find out the effect of Med on lipolysis in different adipocytes. A pterocarpan Med is a natural plant derived compound of Medicago truncatula and Swartzia madagascariensis (13). Previously reported, some beneficial biological functions of Med are improvement of bone regeneration, osteoclastogenesis inhibition, and induction of apoptosis (14-16). Nevertheless, to date, the effects of Med on lipolysis persist to be unknown. We consider that Med can be a candidate of induction of lipolysis and thus reduction of body fat. 


\section{RESULTS}

\section{Effect of Med in cell viability and lipid droplet morphology in BAT cells}

Differentiated brown adipocyte cells were treated with $10 \mu \mathrm{M}$ Med with MDI and cells were harvested to collect protein, mRNA or used for other experiments (Fig. 1A). As shown in Fig. 1B, various concentrations of Med have no cytotoxicity on BAT cells. As shown in Fig. 1C, Med treated BAT cells showed smaller lipid droplets than MDI treated control cells. We have selected and measured 5 biggest LD diameters from each sample and the averages are shown in the Fig. 1D. The average LD diameter (Biggest one's) of MDI sample is almost 6 $\mu \mathrm{m}$ and $\mathrm{MDI} / \mathrm{Med}$ is $1.2 \mu \mathrm{m}$. Data allowed us to speculate whether Med can inhibit of lipid biogenesis or promote lipolysis on BAT cells. In this study, we focused on Med-induced lipolysis on BAT cells.

\section{Med treatment alters the expression of lipid metabolism related genes in BAT cells}

To find out the possible underlying mechanism for smaller lipid droplet formation by Med, we conducted RNA-Seq analysis (Fig. 2A). A total of 3079 genes were found to be differentially expressed by Med treatment (greater than 1.5 fold up and down; Fig. 2B). Among 3079 genes, 814 genes were upregulated more than 2 fold, and 850 genes were down-

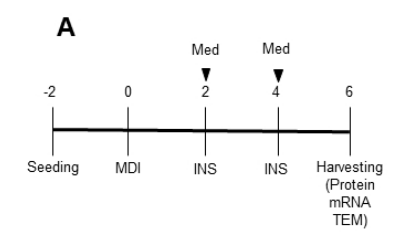

B
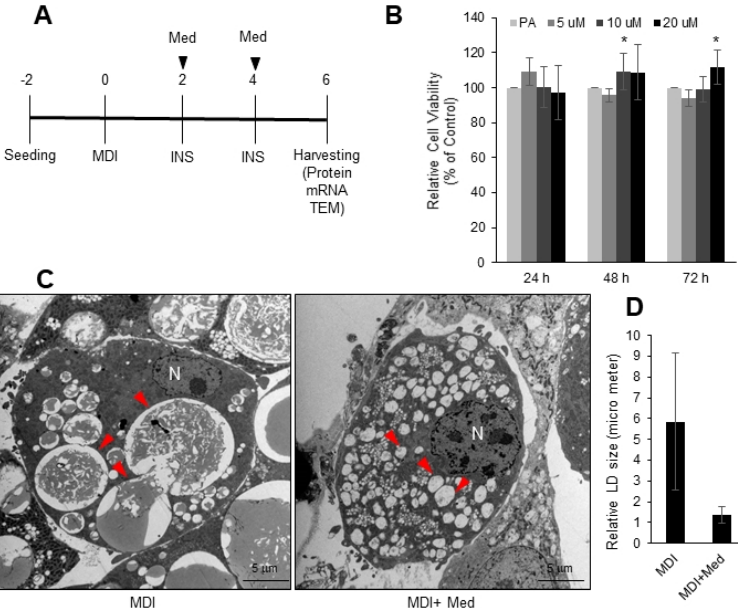

Fig. 1. Effect of Med on lipid droplet morphology in BAT cells. (A) Treatment procedure of Med in BAT cells. (B) Viability of BAT cells was assessed by the MTT assay. Data are expressed as mean \pm SEM of three independent experiments. PA means preadipocyte and Med $5 \mu \mathrm{M}, 10 \mu \mathrm{M}$ and $20 \mu \mathrm{M}$ concentrations are used. PA was used as control and ${ }^{*} P \leq 0.05$. (C) TEM images (3500X magnification) of Med treated BAT cells (Scale bar represents $5 \mu \mathrm{m})$. Red arrows indicate lipid droplets (LD). (D) Relative LD diameter was measured by manual measurement of diameter of 5 biggest LDs from each samples and then calculating their averages. regulated more than 2 fold by Med treated BAT cells. 256 and 260 genes were up and down regulated, respectively by Med treatment. Next, we analyzed G.O categories for those genes and it is shown in Fig. 2C. Among 8 categories, catalytic activity was the highest regulated group (33\%; Fig. 2C). We further scrutinized that expression of 27 genes are altered (greater than log 2 fold up or down) by Med treatment which are involved in the lipid metabolism process (Fig. 2D). From 8 G.O categories we analyzed all the subcategories and found that 4 of those contain DEGs (total 27 genes) that regulate lipid metabolism (Fig. 2D).

\section{Med promotes lipolysis activation related gene expression}

Whole transcriptome shotgun sequencing (RNA-Seq) revealed that Med alters genes expression which are mainly from the PKA regulated pathway and activate lipolysis. As shown in Fig. $3 \mathrm{~A}$, genes involved in regulation of lipolysis are up-regulated by Med treatment with some few genes down-regulation. RNA-seq analysis using iWAT cell samples also showed similar results with lesser extend of upregulation (Sup Fig. S2A). PKA pathway is one of the major regulator of lipolysis (17), and we have found that Med treatment up-regulated the genes from PKA pathway which are directly linked to the process of lipolysis (Pparo, Adrb3, Pnpla2/Atgl, and Hsl; Fig. 3B) (4) although genes that are not related to the lipolysis are found to be down-regulated (Rapgef4, Htr1d, Camk2b, and Adcy1; Fig. 3B). We have confirmed some of those gene expression levels by qRT-PCR (Fig. 3C and D). We also have confirmed some of those lipolysis related gene expression levels in C3H10T1/2 cells (Sup. Fig. S1A) and in iWAT cells (Sup. Fig. S2B). As compared qRT-PCR with RNA-Seq, Med
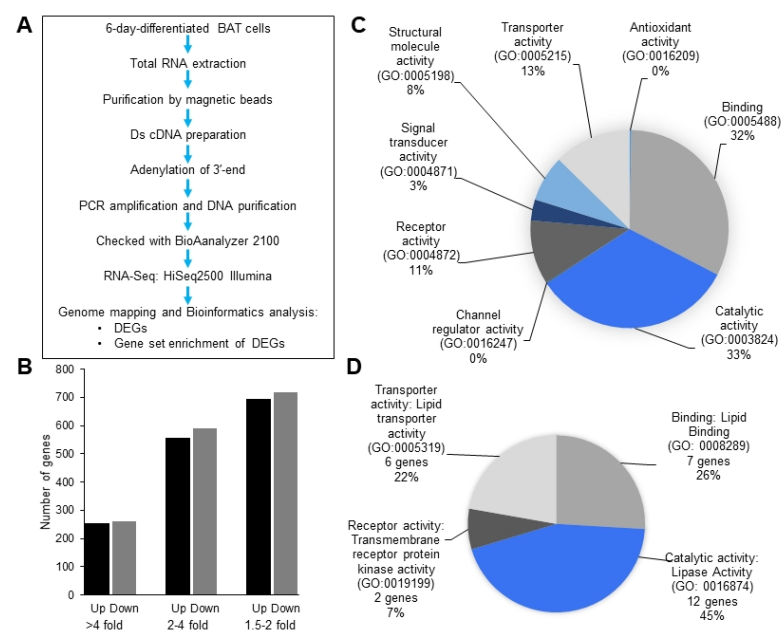

Fig. 2. Med alters the expression of lipolysis regulatory genes in BAT cells. (A) Methods followed for RNA-Seq analysis. (B) Population of DEGs by Med treatment. (C) Pie chart showing G.O categories of DEGs ( $>2$ fold change). (D) Pie chart of G.O categories of DEGs involved in regulation of lipid metabolism. 


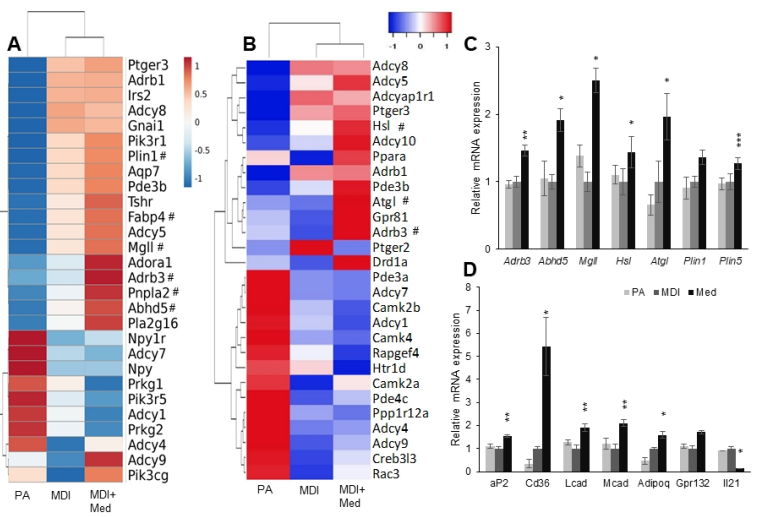

Fig. 3. Med induces differential gene expression of lipolysis-related genes. (A) Heatmap of DEGs of RNA-seq analysis those are involved the regulation of lipolysis. (B) Heatmap of some of the DEGs of RNA-seq analysis from PKA regulated pathway. "Indicates genes of which expression levels were confirmed by qRT-PCR. (C) Some of those DEGs were selected and the expression levels were confirmed by qRT-PCR analysis. (D) Miscellaneous sets of gene expression levels measured by qRT-PCR after Med treatment in BAT cells. Data are stated as mean \pm SEM of three separate experiments. $* P \leq 0.05, * * P \leq 0.01, * * * P \leq 0.001$.

showed similar increase in gene expression of Atgl (1.97 fold, $\mathrm{n}=3), \mathrm{Hsl}$ (1.44 fold), Abhd5 (1.92 fold), Mgll (2.5 fold), and Adrb3 (1.46 fold) (Fig. 3C). We have also found that Med treatment increased gene expression of $\mathrm{aP2}(1.53$ fold, $\mathrm{n}=3)$, Cd36 (5.42 fold), Lcad (1.91 fold), Mcad (2.11 fold), Gpr132 (1.71 fold), and Adipoq (1.59 fold) and reduced the expression of $/ / 21$ ( 0.14 fold) (Fig. 3D). Il21 is reported to be a negative regulator of lipolysis (18). We also have found that Med treatment promotes $\mathrm{Hsl}$ translocation to the lipid droplet body to promote this lipolysis (Sup Fig. S1C).

\section{Activation of PKA is necessary for the Med induced lipolysis in BAT cells}

Next, we tried to find out the possible mechanism of this lipolysis. To do so, we have tested whether Med mediated lipolysis on BAT cells through activation of PKA pathway. As shown Fig. 4B, Med treatment increased phosphorylation of PKA substrates. Med treatment also increased protein expression level of $\mathrm{Hsl}$ and Atgl along with the phosphorylation of $\mathrm{Hsl}$ at the position of Ser ${ }^{660}$, which is a PKA target site (Fig. 4B). Glycerol release from the cells in the media was significantly increased $(120 \%)$ by Med treatment and $165 \%$ by Isoproterenol, respectively (Fig. 4D). We hypothesized that PKA might have role in Med induced lipolysis. To provide insight whether PKA involved in this lipolysis, we performed $P K A-C a t-\alpha 1$ gene silencing of which confirmation is shown in (Fig. 4A). PKA-Cat- $\alpha 1$ gene silencing resulted in the reduction of PKA substrate phosphorylation (Fig. 4B, lane 3). Gene silencing study also revealed that knocking down of PKA-Cat- $\alpha 1$ significantly reduces Hsl phosphorylation (Fig. 4C)

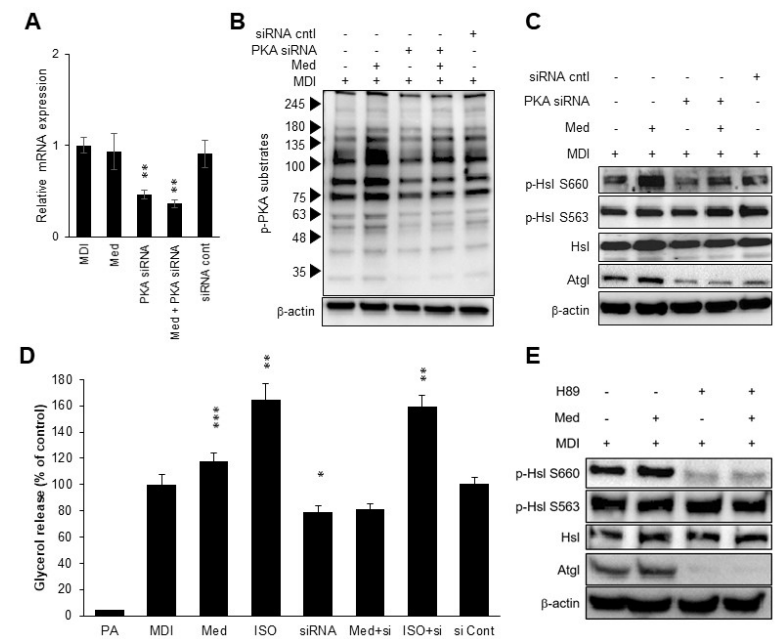

Fig. 4. $P K A-c a t-\alpha 1$ gene silencing abolishes Med induced lipolysis (A) Gene expression of PKA-Cat- $\alpha 1$ to check the efficiency of siRNA experiment. (B) WB images of phospho-PKA substrate and $\beta$-actin. (C) Protein expression levels of Atgl, Hsl, phospho-Hsl $\operatorname{Ser}^{563}$ and $\operatorname{Ser}^{660}$ and $\beta$-actin. Expression of $\beta$-actin was used as an internal control. Data are representative of three independent experiments. (D) Relative percent of glycerol release with control (MDI). Except for PA (pre-adipocytes) all other samples are under $\mathrm{MDI}$ stimulated condition. Data are indicated as mean \pm SEM of three separate experiments. $* P \leq 0.05, * * P \leq 0.01, * * * P \leq$ 0.001 . (E) BAT cells were grown in full confluence and then treated in combination of $\mathrm{H} 89(10 \mu \mathrm{M})$, Med $(10 \mu \mathrm{M})$ and MDI as indicated in the figure. Protein samples were collected at day 6 of differentiation. Protein expression levels of Atgl, $\mathrm{Hsl}$, phospho-HsI $\operatorname{Ser}^{563}$ and $\operatorname{Ser}^{660}$ and $\beta$-actin were analyzed by WB. Expression of $\beta$-actin was used as an internal control. Data are representative of three independent experiments.

as well as glycerol release (Fig. 4D). Med treatment couldn't recover the process.

To test effect of the inhibition all types of PKA isoforms we performed PKA pan inhibition by H89. And data revealed that inhibition of PKA by $\mathrm{H} 89$ also reduced $\mathrm{Hsl}$ phosphorylation at $\mathrm{Ser}^{660}$ and expression of Atgl protein. Data reveal that the activation of PKA is necessary for the Med mediated lipolysis in BAT cells.

\section{DISCUSSION}

The major findings of this study are Med can upregulate the expression of lipolysis related genes and promote glycerol release from BAT cells. Next, Med also promotes PKA substrate phosphorylation and induces $\mathrm{Hsl}$ phosphorylation at PKA target position. In addition, Med promotes differential gene expression of lipolysis related genes both in iWAT and C3H10T1/2 cells (Sup. Fig. S1A, S2A and S2B). Furthermore, Med triggers the translocation of $\mathrm{Hsl}$ from cytosol to lipid droplet membrane in $\mathrm{C} 3 \mathrm{H} 10 \mathrm{~T} 1 / 2$ cells (Sup Fig. S1C). Finally, the Med mediated lipolysis and Hsl-phosphorylation are 
inhibited by the PKA-cat- $\alpha 1$ gene silencing, indicating that these effects are mediated by a PKA-dependent pathway.

Bukowiecki et al., showed that lipolysis and respiration are strongly correlated in brown adipocytes (8). They found that inhibition of lipolysis in brown adipocytes reduces respiration or vice versa (8). We have found increased lipolysis as well as increased beta oxidation regulatory genes Mcad and Lcad expression by Med treatment which might lead to further investigation of the relationship between lipolysis and respiration in BAT cells. Another study has showed that for the heat production to defend the body against cold, brown adipose tissue-mediated triglyceride clearance and burning of fatty acids are necessary (9).

The RNA-Seq analysis has revealed that Med differentially expresses lipolysis regulatory gene profiles in BAT cells and we sought to confirm that findings by using different experimental techniques. This RNA-Seq analysis has also indicated that PKA signaling pathway might be the regulatory hub for the Med-induced lipolysis. cAMP/PKA/Hsl signal axis is one of the best known mechanisms among the pathways that regulate lipolysis in the adipocytes (19). In this pathway, external stimuli input signal via beta adrenergic receptor to increase the activity of adenylyl cyclases and CAMP production which can activate PKA signaling. PKA then phosphorylates $\mathrm{Hsl}$ and perilipin which regulate lipolysis (19). Here, we demonstrated that Med stimulates basal lipolysis in cultures of murine adipocytes and it is perceptible that this Med-induced lipolysis is mediated by $\mathrm{Hsl}$ activation via classic cAMP and PKA signaling, because we observed increase in Adrb3 gene expression, phosphorylation of PKA substrates as well as $\mathrm{Hsl}$ phosphorylation at PKA target position in BAT cells. Moreover, an increase in isoproterenol-stimulated lipolysis (Glycerol release) in the Med-treated C3H10T1/2 cells (Sup. Fig. S1B). Atgl and $\mathrm{Hsl}$ are the major lipases that regulate lipolysis and they are activated mainly by phosphorylation (4). In the process of lipolysis, PKA not only phosphorylates Hsl, Atgl but also perilipin (19). Although we detected Hsl phosphorylation (at $\mathrm{Ser}^{660}$ but not in $\mathrm{Ser}^{563}$ ) and translocation in LD surface, we could not detect Atgl and perilipin phosphorylation. We also observed increased Atgl and Hsl gene and protein expression levels in Med-treated cultures of BAT and $\mathrm{C} 3 \mathrm{H} 10 \mathrm{~T} 1 / 2$ cells which correlate with previous study of lipolysis induction (20).

Here, we demonstrated that the PKA regulated pathway is mainly responsible for lipolysis by Med, but we cannot exclude contribution of other signaling pathways for lipolysis from our result whereas PKA gene silencing reduced most of the $\mathrm{Hsl}$ phosphorylation (Fig. 4B), lipolysis was not ablated completely (Fig. 4C). Since gene silencing of other pathways were not applied in this study, the activation of lipolysis by Med might be regulated in parallel by some other signals. However, additional study is necessary to elucidate the other mechanisms involved in the lipolytic activity of Med on BAT cells. Further in vivo studies are mandatory to scrutinize the
Med induced lipolysis and define whether lipolytic enzyme gene expression and direct activation of $\mathrm{Hsl}$ by Med are correlated to the enhanced lipid degradation in response to $\beta$-adrenergic signals. In summary, pterocarpan Med effectively promotes lipolysis via alteration of lipolytic-related gene expression specially cAMP/PKA/Hsl axis on BAT cells.

\section{MATERIALS AND METHODS}

\section{Chemicals, reagents and antibodies}

Medicarpin (purity 98\%, HPLC) was purchased from ChemFaces Biochemical Co., Ltd. (Wuhan, Hubei, China). Dexamethasone, Insulin, isobutyl-1-metylxanthine (IBMX), 3-(4,5-dimethylthiazol-2-yl)-2,5-diphenyltetrazolium bromide (MTT), Isoproterenol (Iso) and rosiglitazone (Rosi) were purchased from Sigma-Aldrich (St. Louis, MO, USA). Fetal bovine serum (FBS) and High-glucose Dulbecco's modified Eagle medium (DMEM) were obtained from Atlas Biologicals. Penicillin-streptomycin solution was obtained from Hyclone Laboratories, Inc. (South Logan, NY, USA). Antibodies against HSL, Phospho-HSL (Serine 563 and 660), ATGL, Phospho-PKA substrate and Perilipin were purchased from Cell Signaling Technology (Danvers, MA, USA). Antibody against $\beta$-actin was purchased from Abcam (Cambridge, MA, USA). BCA protein assay kit was purchased from Thermo Scientific (Rockford, IL, USA) and protein loading buffer was obtained from Bio-Rad Laboratories, Inc. (Hercules, CA, USA). Bodipy 493/503 was obtained from Life Technologies Corporation (Carlsbad, CA, USA).

\section{Cell culture and differentiation}

BAT cells (Kind Donation of GE Kai, Senior Investigator, NIH), C3H10T1/2 MSCs (Korean Cell Line Bank, KCLB-10226) and iWAT (Kind donation of Yun-Hee Lee, Yonsei University) were cultured and maintained in DMEM Glutamax media supplemented with $10 \%$ FBS and $1 \%$ Penicillin-Streptomycin and were kept at $37^{\circ} \mathrm{C}$ in $5 \% \mathrm{CO}_{2}$ incubator. BAT and iWAT are immortalized cell lines developed by individual scientist. The cells were differentiated as described elsewhere $(21,22)$. Cells after 6 days of differentiation were used in all experiments.

\section{Cell treatment}

Differentiated cells were treated with or without Med (10 $\mu \mathrm{M})$ and rosiglitazone $(1 \mu \mathrm{M})$ in differentiation medium at day 4 and harvested at day 6 . Media were changed every other day. Preadipocytes (PA) were maintained only in culture media (DMEM and FBS). Isoproterenol $(10 \mu \mathrm{M})$ and rosiglitazone (1 $\mu \mathrm{M})$ were used as positive control as indicated in figures.

\section{Cell viability assay}

BAT cells were seeded in 96-well plates at a density cells become 80 to $90 \%$ confluent. Cell viability using MTT assay was performed as described elsewhere (23). 


\section{Gene silencing analyses}

BAT cells were transfected with a PKA-cat- $\alpha 1$ siRNA (Santa Cruz Biotechnology, Inc.) oligonucleotide duplex at $80 \%$ to $90 \%$ confluence with Lipofectamine 2000 by following the manufacturer's protocol. Briefly, 100 pM siRNA was transfected with $6 \mu \mathrm{l} /$ well Lipofectamine 2000 in four 6-well plates. Lipofectamine 2000 and siRNA were individually diluted in $100 \mu \mathrm{l}$ of the Opti-MEM medium (incubated for $5 \mathrm{~min}$ ), mixed, incubated for $20 \mathrm{~min}$ at room temperature, and then added into each well. The medium was removed and replaced with the induction medium (MDI) after 100\% confluence in the presence or absence of Med. The efficiency of siRNA silencing was determined by WB after 24 hours of transfection. Protein and RNA were collected on day 8 of cell differentiation and subjected to western blotting and qRT-PCR for the analysis of downstream genes.

\section{Bodipy 493/503 lipid staining with Perilipin, HsI immunostaining}

8 days differentiated $\mathrm{C} 3 \mathrm{H} 10 \mathrm{~T} 1 / 2$ MSCs were stained with Bodipy 493/503 lipid staining with Perilipin and $\mathrm{Hsl}$ antibodies as described elsewhere (23).

\section{Quantitative RT-PCR and Whole-Cell extracts preparation for Westem Blot analyses}

Total RNA extract and qRT-PCR using BAT, iWAT and $\mathrm{C} 3 \mathrm{H} 10 \mathrm{~T} 1 / 2$ cells were performed as described before (24). The sequences for the primers used in this study are listed in Supplementary Table 1. The target gene expression was normalized to that of ribosomal protein large P0 subunit $(P O)$. Whole-cell extracts preparation using BAT cells for western blot (WB) analysis was performed as described elsewhere (25). Some changes were made as, addition of phosphatase inhibitor (Sigma-Aldrich St. Louis, MO, USA) into RIPA lysis buffer cocktail and replacement of skim milk for blocking of membrane with $1 \%$ BSA in case of phospho proteins WB.

\section{Sample preparation and RNA-Seq analyses}

Total RNA was extracted from each sample as described elsewhere (26). An RNA-Sequencing library was generated using Nextflex Rapid Directional qRNA-Seq Kit according to user's instruction manual (Bioo Scientific, TX, USA). Briefly, mRNA was purified from total RNA using Oligo (dT) beads and fragmented chemically. After double-strand cDNA synthesis of the fragmented mRNA, adenylation of 3'-end, sequencing adapter ligation, UDG treatment and PCR amplification were performed, followed by DNA purification with magnetic beads. Finally, the amplified library was checked with BioAanalyzer 2100 (Agilent, CA, USA), and then applied for sequencing template preparation. The HiSeq2500 platform was utilized to generate 100-bp paired-end sequencing reads (Illumina, CA, USA). RNA-seq data were deposited on NCBI SRA site (https://www.ncbi.nlm.nih.gov/sra) under
SRR5451352， SRR5451351， SRR5451350， SRR5451349， SRR5451348, SRR5451347 numbers.

\section{Genome mapping and Bioinformatics analysis}

The quality of the reads was checked using FastQC (v0.11.5) and trimming low-quality bases and adaptor sequences were performed using cutadapt (v1.11) and trim galore (v0.4.4) respectively. High quality reads were aligned to the mouse reference $(\mathrm{mm} 10)$ by STAR (v2.5.2b). Mapping data was imported into StrandNGS v2.9 (Strand Genomics, CA, USA) for gene counting and differential expression analysis. For gene set enrichment of differentially expressed genes, metascape (www.metascape.com) was used.

\section{Lipolysis assay}

Glycerol release assay (Sigma-Aldrich, St. Louis, MO, USA) for the determination of lipolysis in fully differentiated BAT and C3H10T1/2 cells were performed as described elsewhere (24).

\section{Transmission electron microscopy (TEM)}

TEM experiments were performed as described elsewhere (9). The instruments used in this study are, TEM: H-7000, Htachi, Japan. And Ultra microtome: Ultracut-S Leica, Germany.

\section{Statistics}

All data represented in this experiment are as mean \pm standard error of the mean (SEM) of three or more independent experiments as mentioned in each figure legend. MDI treated groups were used as control to measure fold change unless otherwise stated in the figure legend. Student's t-test was used to determine significant differences between control groups and different treatment groups. ${ }^{*} \mathrm{P} \leq 0.05,{ }^{*} * \mathrm{P} \leq 0.01, * * * \mathrm{P}$ $\leq 0.001$. A P value of $<0.05$ was considered statistically significant.

\section{ACKNOWLEDGEMENTS}

This research was supported by Basic Science Research Program through the National Research Foundation of Korea (NRF) funded by the Ministry of Education (2015R1A 6A103032522), and partially by a research fund of Soonchunhyang University.

\section{CONFLICTS OF INTEREST}

The authors have no conflicting interests.

\section{REFERENCES}

1. Lass A, Zimmermann R, Oberer M and Zechner R (2011) Lipolysis-a highly regulated multi-enzyme complex mediates the catabolism of cellular fat stores. Prog Lipid Res 50, 14-27

2. Klebanov S, Astle CM, DeSimone O, Ablamunits $\mathrm{V}$ and 
Harrison DE (2005) Adipose tissue transplantation protects $\mathrm{ob} / \mathrm{ob}$ mice from obesity, normalizes insulin sensitivity and restores fertility. J Endocrinol 186, 203-211

3. Greenberg AS, Shen WJ, Muliro K et al (2001) Stimulation of lipolysis and hormone-sensitive lipase via the extracellular signal-regulated kinase pathway. J Biol Chem 276, 45456-45461

4. Frühbeck G, Méndez-Giménez L, Fernández-Formoso JA, Fernández S and Rodríguez A (2014) Regulation of adipocyte lipolysis. Nutr Res Rev 27, 63-93

5. Cannon B and Nedergaard JAN (2004) Brown adipose tissue: function and physiological significance. Physiol Rev 84, 277-359

6. Nedergaard J, Bengtsson T and Cannon B (2007) Unexpected evidence for active brown adipose tissue in adult humans. Am J Physiol Metab 293, E444-E452

7. Locke RM, Rial E, Scott ID and Nicholls DG (1982) Fatty acids as acute regulators of the proton conductance of hamster brown-fat mitochondria. FEBS J 129, 373-380

8. Bukowiecki LJ, Follea N, Lupien J and Paradis A (1981) Metabolic relationships between lipolysis and respiration in rat brown adipocytes. The role of long chain fatty acids as regulators of mitochondrial respiration and feedback inhibitors of lipolysis. J Biol Chem 256, 12840-12848

9. Bartelt A, Bruns OT, Reimer R et al (2011) Brown adipose tissue activity controls triglyceride clearance. Nat Med 17, 200-205

10. Chen W, Zhang Q, Cheng S, Huang J, Diao G and Han J (2017) Atgl gene deletion predisposes to proximal tubule damage by impairing the fatty acid metabolism. Biochem Biophys Res Commun 487, 160-166

11. Ogasawara J, Nomura S, Rahman N et al (2010) Hormone-sensitive lipase is critical mediators of acute exercise-induced regulation of lipolysis in rat adipocytes. Biochem Biophys Res Commun 400, 134-139

12. Sztalryd C, Xu G, Dorward H et al (2003) Perilipin A is essential for the translocation of hormone-sensitive lipase during lipolytic activation. J Cell Biol 161, 1093-1103

13. Fedoreyev SA, Pokushalova TV, Veselova MV et al (2000) Isoflavonoid production by callus cultures of Maackia amurensis. Fitoterapia 71, 365-372

14. Dixit M, Raghuvanshi A, Gupta CP et al (2015) medicarpin, a natural pterocarpan, heals cortical Bone defect by activation of Notch and Wnt canonical signaling pathways. PLoS One 10, e0144541
15. Gatouillat G, Magid AA, Bertin E et al (2015) Medicarpin and millepurpan, two flavonoids isolated from Medicago sativa, induce apoptosis and overcome multidrug resistance in leukemia P388 cells. Phytomedicine 22, 1186-1194

16. Tyagi AM, Gautam AK, Kumar A et al (2010) Medicarpin inhibits osteoclastogenesis and has nonestrogenic bone conserving effect in ovariectomized mice. Mol Cell Endocrinol 325, 101-109

17. Seino S and Shibasaki T (2005) PKA-dependent and PKA-independent pathways for cAMP-regulated exocytosis. Physiol Rev 85, 1303-1342

18. Fabrizi M, Marchetti V, Mavilio M et al (2014) IL-21 is a major negative regulator of IRF4-dependent lipolysis affecting Tregs in adipose tissue and systemic insulin sensitivity. Diabetes 63, 2086-2096

19. Carmen G-Y and Víctor SM (2006) Signalling mechanisms regulating lipolysis. Cell Signal 18, 401-408

20. $\mathrm{Xu} \mathrm{C}$, He J, Jiang $\mathrm{H}$ et al (2009) Direct effect of glucocorticoids on lipolysis in adipocytes. Mol Endocrinol 23, 1161-1170

21. Rosenwald M, Perdikari A, Weber E and Wolfrum C (2013) Phenotypic analysis of BAT versus WAT differentiation. Curr Protoc Mouse Biol 205-216

22. Rosen ED and MacDougald OA (2006) Adipocyte differentiation from the inside out. Nat Rev Mol Cell Biol 7, 885-896

23. Imran KM, Rahman N, Yoon D, Jeon M, Lee BT and Kim YS (2017) Cryptotanshinone promotes commitment to the brown adipocyte lineage and mitochondrial biogenesis in C3H10T1/2 mesenchymal stem cells via AMPK and p38-MAPK signaling. Biochim Biophys Acta 1862, 1110-1120

24. Rahman N, Jeon M and Kim YS (2016) Delphinidin, a major anthocyanin, inhibits 3T3-L1 pre-adipocyte differentiation through activation of $\mathrm{Wnt} /$ beta-catenin signaling. Biofactors 42, 49-59

25. Jeon M, Rahman N and Kim YS (2016) Wnt/B-catenin signaling plays a distinct role in methyl gallate-mediated inhibition of adipogenesis. Biochem Biophys Res Commun $479,22-27$

26. Imran KM, Yoon D and Kim YS (2017) A pivotal role of AMPK signaling in medicarpin-mediated formation of brown and beige adipocytes from C3H10T1/2 mesenchymal stem cells. BioFactors, 44, 168-179 